\title{
Low Energy TEM Characterizations of Ordered Mesoporous Silica-Based Nanocomposite Materials for Catalytic Applications
}

\author{
Jafar F. Al-Sharab ${ }^{*}, 1$, Eliška Mikmeková ${ }^{2}$, Sayantani Das $^{3}$, Anandarup Goswami $^{3,4}$, Said M. El-Sheikh ${ }^{5}$, \\ Adel A. Ismail ${ }^{5}$, Mahdi Hesari ${ }^{6}$, Flavio Maran ${ }^{6}$, and Tewodros Asefa ${ }^{3,4}$ \\ 1. Department of Materials Science and Engineering, Rutgers University, Piscataway NJ, USA \\ 2. Institute of Scientific Instruments of the ASCR, v.v.i., Kralovopolska 147, Brno 612 64, Czech \\ Republic \\ 3. Department of Chemistry and Chemical Biology, Rutgers University, Piscataway NJ, USA \\ 4. Department of Chemical and Biochemical Engineering, Rutgers University, Piscataway NJ, USA \\ 5. Nanostructured Materials and Nanotechnology Division, Advanced Materials Department, Central \\ Metallurgical Research and Development Institute (CMRDI), Helwan 11421, Cairo, Egypt \\ ${ }^{6 .}$ Department of Chemistry, University of Padova, 35131 Padova, Italy \\ ${ }^{*}$ Corresponding author. Email:jafarhan@rci.rutgers.edu
}

Highly ordered mesoporous SBA-15-based silica nanocomposite materials are receiving a great deal of attention due to their interesting structure, which allows hosting nanoparticles for catalytic applications, among many other things. However, to maximize catalytic efficiency, understanding the relationship between their structures and surface chemistries as well as different properties at the subnanometer scale is required. This is essential in order to correlate their structure with their properties and, thereby, develop materials with the best performance. However, such studies are sometimes limited by the lack of reliable characterization techniques, particularly those offering minimal induced damage to the material while allowing detailed analysis of its microstructural features. Therefore, development of such techniques is essential for the overall improvement of catalytic materials.

Many of the current characterization techniques, especially those utilizing high energetic beams, induce damage in samples via several mechanisms, leaving precise quantification questionable. For example, recent studies have shown that improper data collection of fluoride-based electrodes will severely damage samples and cause beam-induced phase transformation [1] in very short exposure time $(\sim 1 \mathrm{~ms})$. Under extreme conditions evidence of sublimation of the irradiated area may also occur [2].

In this work, we synthesized highly ordered mesoporous silica structures (SBA-15) with uniform and closed packed pores of $\sim 7 \mathrm{~nm}$ internal diameter. The internal channels were decorated with nano size precious metal such as $\mathrm{Au}, \mathrm{Pt}$, and $\mathrm{Pd}$. When particle size is greater than pore size, nanoparticles are also deposited on the matrix surface. Size of the active catalytic sites varies from ultra-small $(<2 \mathrm{~nm})$ to relatively large $(<30 \mathrm{~nm})$, depending on synthesis and deposition technique. These structures show very promising results as catalytic systems due to high surface area and density of catalytic active sites. Detailed electron microscopy characterization techniques including scanning and transmission electron microscopy techniques (or their combination - scanning transmission EM) are employed to characterize these structures at the atomic scale. The effect of beam energy on the radiation damage is also being studied.

SBA-15 template was synthesized following standard techniques. Au, Pd, and Pt were then deposited on SBA-15 templates, Fig. 1, following two different protocols. Detailed information regarding synthesis, 
and catalytic activity can be found elsewhere [3,4]. All synthesized samples have been studied using microscopy and spectroscopy techniques with beam energy varying from $600 \mathrm{eV}$ to $200 \mathrm{KeV}$ in order to properly characterize samples and to understand the effect of electron beam on the structure of the SBA-15-based nanocomposites. Scanning Low Electron Energy Microscopy (SLEEM) equipped with Cathedo lens was utilized to observe samples' surface at low accelerating voltage. JEOL2010F TEM equipped with EDS and EELS was utilized to obtain chemical mapping with probe size of $1 \AA$ and enough energy for chemical analyses. All EELS and EDS spectra were automatically drift corrected. Fig. 2- a.) compares standard SEM and SLEEM observations of SBA-SH-Au-144- at 10keV, and 0.6 $\mathrm{keV}$. STEM observations of Au nanoparticles in bright field (BF), dark field (DF) and high angular annular dark field (HAADF) modes (Magellan 400, FEI) for identical areas is presented in Fig. 2- b.). The low energy electron microscopy technique shows significant improvement in minimizing the irradiation damage and the interaction volume of the studied samples, and hence, accurate quantification of these nanocomposites.

This work is the first step to establish a protocol optimizing the characteristics of the electron beam and collection conditions liable to cause no or minimal radiation damage of SBA-15-based nanocomposites. The effect of sample's holder temperature on collection conditions is also being investigated. We expect that these results will pave the road for precise quantification of mesoporous silica (SBA-, and MCM-based) and various nanostructured materials.

\section{References:}

[1] J. F. Al-Sharab et al., Journal of Nanoparticle Research, Volume 15, issue 4 (April 2013), p. 1-12. ISSN: 1388-0764 DOI: 10.1007/s11051-013-1500-1.

[2] J. F. Al-Sharab et al., Cryst. Growth Des., 2009, 9 (11), pp 4680-4684, DOI: 10.1021/cg900544k

[3] Das, S. et al., (2014), Small. doi: 10.1002/smll.201302854.

[4] El-Sheikh, S. M. et al., New Journal of Chemistry, Volume 37, issue 8 (July 15, 2013), p. 2399-2407, ISSN: 1144-0546 DOI: 10.1039/c3nj00138e.

[5] Low energy electron microscopy was conducted at the Institute of Scientific Instruments of the ASCR, v.v.i., Kralovopolska 147, Brno 612 64, Czech Republic.
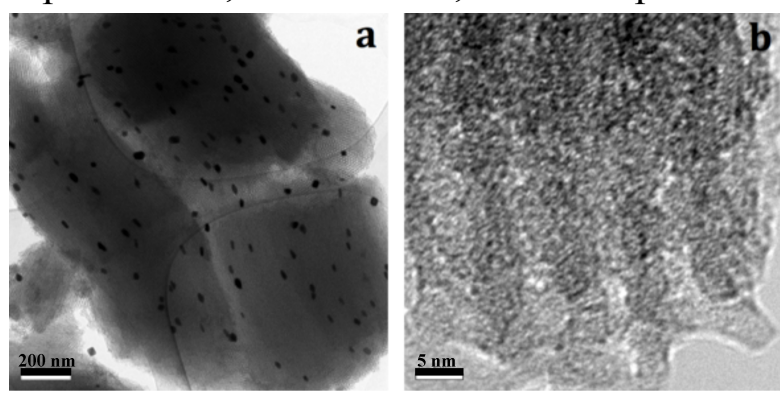

Figure 1. SBA-15 matrix decorated with (a) Pt and (b) Au nanoparticles.

(a)

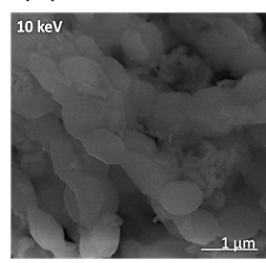

(b)

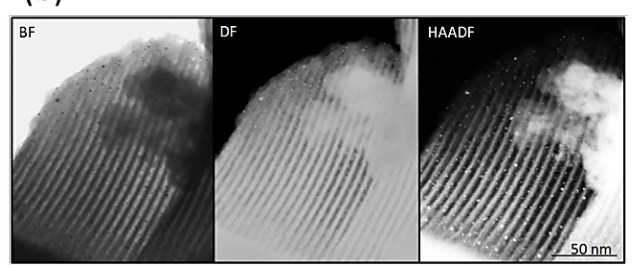

Figure 2. (a) SLEEM observation of SBA-SH-Au-144-5 at $10 \mathrm{keV}$ and $0.6 \mathrm{keV}$. (b) STEM observation of SBA-SH-Au-144-5 in BF, DF and HAADF modes at $30 \mathrm{keV}$ 